ORIGINAL ARTICLE

\title{
Echocardiographic abnormalities in primary antibody deficiency
}

\author{
S L Johnston, S J Hill, R J Lock, J F Dwight, D J Unsworth, M M Gompels
}

Postgrad Med J 2004;80:214-218. doi: 10.1136/pgmi.2003.012443

See end of article for authors' affiliations

....................

Correspondence to: Dr Sarah L Johnston, Department of Immunology and Immunogenetics, Southmead Hospital Westbury-on-Trym, Bristol BS10 5NB, UK; sljoh@ hotmail.com

Submitted 11 July 2003 Accepted

8 September 2003
Objective: To document cardiac abnormalities secondary to pulmonary disease in primary antibody deficiency.

Patients and methods: A cross sectional audit study of patients from a regional immunology centre. Subjects undergoing two dimensional and Doppler transthoracic echocardiography were reviewed. Ventricular dimensions and function, valvular competence, and estimated pulmonary artery pressure were recorded. Data were compared with clinical variables, pulmonary function tests, and thoracic computed tomography data.

Results: Nineteen patients with common variable immunodeficiency and one with $\lg _{2}$ subclass deficiency were included, mean age at diagnosis 37.5 years, mean estimated diagnostic delay 10.94 years. Left ventricular impairment was found in $15 \%$ and right heart dilatation in $20 \%$. Pulmonary hypertension (mean pulmonary artery pressure $>25 \mathrm{~mm} \mathrm{Hg}$ ) was found in $45 \%(9 / 20)$, graded as moderate (40$60 \mathrm{~mm} \mathrm{Hg}$ ) in $44 \%$ of cases. Pulmonary function was obstructive in $47 \%(9 / 19)$. Fifty five percent of the patients with computed tomography data within the last five years (10/18) had confirmed bronchiectasis. Patients with right heart dilatation and/or moderate pulmonary hypertension $(n=6)$ had a more prolonged diagnostic delay $(p=0.04)$ and more severe lung disease.

Conclusion: Echocardiographic abnormalities are common in primary antibody deficiency, associated with diagnostic delay and pulmonary complications. Pulmonary hypertension should be considered in those with severe lung disease and can be confirmed by echocardiography.
$\mathrm{P}$ rimary antibody deficiency syndromes are uncommon and often poorly recognised. ${ }^{1}$ Patients present with recurrent upper and lower respiratory tract infections with consequent chronic pulmonary damage and the development of bronchiectasis (fig 1). Primary antibody deficiency syndromes can be subdivided into several distinct disease categories of which common variable immunodeficiency (CVID) is the most frequent, with an estimated incidence of one in 10000 to one in $50000 .^{2}$ CVID is characterised by defective antibody formation accompanied by decreased serum IgG and IgA levels with or without IgM deficiency. Early recognition and adequate immunoglobulin replacement treatment aims to reduce the infection rate and thereby avoid pulmonary complications.

An audit of patients with antibody deficiency in the UK from 1993-96 (including 686 patients with CVID) found that the diagnostic delay for CVID was 7.3 years. Nearly $20 \%$ had bronchiectasis, which in most cases was established before the patient was first seen by an immunologist. ${ }^{1}$ Cardiac complications secondary to underlying chronic pulmonary disease have not been previously documented in this patient population. Dyspnoea secondary to poor cardiac function may be ascribed to poor pulmonary function and therefore not addressed adequately. By analogy to patients with cystic fibrosis complicated by bronchiectasis where cardiac manifestations are well documented, ${ }^{3-5}$ right sided heart disease was postulated to occur in antibody deficient patients with the most severe pulmonary disease.

\section{METHODS}

Twenty adult patients with primary antibody deficiency under the care of a regional immunology centre underwent two dimensional and Doppler transthoracic echocardiography as part of their routine follow up. Inclusion criteria for the study were a confirmed diagnosis of primary antibody deficiency and ongoing immunoglobulin replacement therapy. Patients were excluded if they had a history of preexisting cardiac disease. All echocardiograms were performed using an ATL (Hdi) ultrasound machine with the Harmonic Imaging option and $3.5 \mathrm{MHz}$ probe, between May and December 2001. The cardiology technician (SJH) was blinded to the patient's clinical status. Standard views were used to assess function and size of the cardiac chambers as well as valve competence and patency. The extracardiac space was assessed using the subcostal view. Normal values were taken from Walsh and Wilde ${ }^{6}$; these are used in most cardiac centres in the UK. Estimated pulmonary artery pressure was derived from the amount of tricuspid regurgitation using the modified Bernoulli equation, in addition to the estimated right atrial pressure. Pulmonary artery pressure was considered normal up to $25 \mathrm{~mm} \mathrm{Hg}$ and pulmonary hypertension was defined as mild $(25-40 \mathrm{~mm} \mathrm{Hg})$, moderate (40$60 \mathrm{~mm} \mathrm{Hg}$ ), or severe (>60 mm Hg).

Information relating to age, diagnostic delay, time on replacement therapy before echocardiography, pulmonary function, and thoracic imaging was collected and analysed retrospectively.

Formal pulmonary function tests were performed through the respiratory medicine department at the regional centre. The Morgan Benchmark TT501 system was used after October 1999 for flow volume loops and spirometry, before this the Micro-Medical system was in use, with spirometry on a standard vitallograph spirometer. Oxygen saturations were recorded using an Omeda 3700e pulse oximeter and blood gas analysis was performed on a Rapid lab 248 (Chiron Diagnostics). Standard European pulmonary function

Abbreviations: CVID, common variable immunodeficiency; $\mathrm{FEV}_{1} / \mathrm{FVC}$, forced expiratory volume in one second/forced vital capacity 


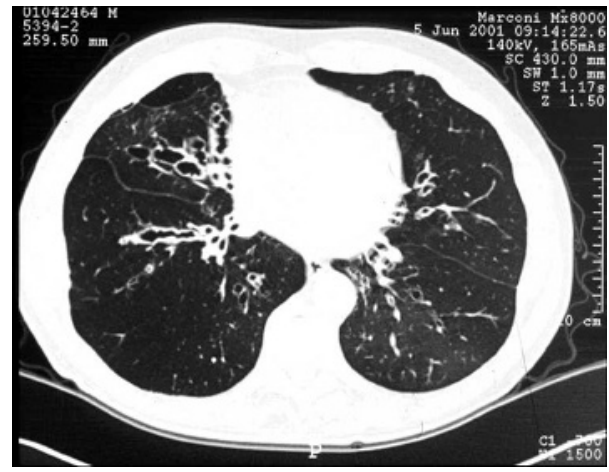

Figure 1 Thoracic computed tomogram demonstrating extensive bronchiectasis throughout both lungs. The airways are markedly dilated with both cylindrical and saccular changes.

reference ranges according to age, height, and gender were employed. ${ }^{7}$

High resolution thoracic computed tomography was performed on an El Scint twin spiral scanner before 2000, with subsequent scans on a Marconi Multispiral system via the radiology department at the regional centre $(n=17)$. Two had high resolution scans at outreach hospitals and one had a conventional scan at the regional centre. Films were reviewed by a single radiologist in 17 of 18 cases and previous scans were compared where available.

It is our routine practice to perform baseline thoracic computed tomography at diagnosis and, at the time of the study, at five yearly intervals thereafter. We aim to perform pulmonary function tests at two yearly intervals and maintain trough IgG levels (IgG just before the next infusion) at $8 \mathrm{~g} / \mathrm{l}$ or above (see discussion).

As all investigations were part of the patients' routine follow up (an audit of care) ethics committee approval was not required.

Statistical analysis was performed using Fisher's exact test, Mann-Whitney U test or $\chi^{2}$ test as appropriate, comparing those with right heart dilatation and/or moderate pulmonary hypertension to those without.

\section{RESULTS}

Twenty patients were included in the study, 19 with CVID and one with $\operatorname{IgG}_{2}$ subclass deficiency. Demographic data are set out in table 1 . The mean age of the patient cohort was 48.9 years, range 20.5-77.25 years. Mean age at diagnosis was 37.5 years, range 15.3-68.8 years, data not shown. The mean delay in diagnosis, defined as the delay between the onset of recurrent infection and recognition of antibody deficiency, as based on the patients' history, could be estimated in 17/20, and was 10.94 years, range 3-35 years. This delay was most marked in those patients with right heart dilatation and/or at least moderate pulmonary hypertension at 16.3 years, compared with eight years in those without such abnormalities. The correlation between diagnostic delay and right heart abnormalities was significant, $\mathrm{p}=0.04$. There was a trend towards a positive correlation between time from symptom onset and echo abnormalities, however this did not reach significance; echo abnormalities therefore were not simply a function of disease duration.

The echocardiographic findings are summarised in table 2 . Left ventricular impairment was identified in $15 \%$ (patients 1,6 , and 16), all of whom had bronchiectasis and evidence of arterial hypoxaemia. Left ventricular hypertrophy was identified in $20 \%(4 / 20), 1 / 4$ with known systemic hypertension. Aortic root dilatation was seen in $15 \%$ (patients 1,6 , and 12 ). Mitral regurgitation of any degree was found in 65\% (13/20) but was thought to be trivial and insignificant in all but two patients. Tricuspid regurgitation of any degree was found in $90 \%(18 / 20)$ but again thought to be trivial and insignificant in all but three patients. No significant pulmonary regurgitation was identified and the aortic valve was competent in all cases. Right heart dilatation was found in $20 \%$ (patients 2, 3 , 4, and 6). Pulmonary hypertension, estimated pulmonary artery pressure $>25 \mathrm{~mm} \mathrm{Hg}$ was found in $45 \%$ (9/20). This was mild in 5/9, only one of these five having a pressure above $30 \mathrm{~mm} \mathrm{Hg}$ (patient 16). Moderate pulmonary hypertension occurred in 4 (patients 1, 3, 5, and 6), with a maximum estimated pressure of $48 \mathrm{~mm} \mathrm{Hg}$.

Table 3 documents pulmonary function results and findings on thoracic computed tomography. Spirometry was obstructive (forced expiratory volume in one second/forced vital capacity $\left.\left(\mathrm{FEV}_{1} / \mathrm{FVC}\right)<70 \%\right)$ in $47 \%$ (9/19), patient 16 was unable to perform the test because of dyspnoea. However formal pulmonary function data within the last two years was only available for $31.5 \%$ (6/19). Spirometry was previously normal in 9/13 where data were more than two years old. Hypoxia was recorded by oximetry (oxygen saturation $<93 \%$ on room air) in two cases and arterial hypoxaemia (arterial oxygen pressure $<10.5 \mathrm{kPa}$ ) was confirmed by blood gas analysis in five. These two groups were not overlapping. Fifty five percent of patients with a thoracic computed tomogram within the last five years (10/18) had documented bronchiectasis. This was stable, single lobe in $10 \%$, progressive single lobe in $10 \%$, mild multilobar in $50 \%$, and severe multilobar in 30\% (patients 1, 2, and 6). Patient 7 had had a lobectomy for bronchiectasis before a diagnosis of antibody deficiency, bringing the total with documented bronchiectasis to $60 \%$.

For statistical analysis, oxygen status was defined as abnormal if oxygen saturations were less than $93 \%$ or arterial oxygen pressure $<10.5 \mathrm{kPa}$ on room air. Thoracic computed tomography results were divided into three categories: normal/no significant bronchiectasis (patients 5, 10, 17, 18, 19); mild/moderate abnormality (patients 7, 8, 9, 11, 12, 14, 15); and severe with or without emphysema (patients 1, 2, 3, $4,6,13,16,20)$. The correlation between computed tomography and echo abnormalities was significant, $\mathrm{p}=0.03$.

The 30\% (6/20) with evidence of right heart dilatation and/ or moderate pulmonary hypertension (defined as having right heart abnormalities) were those with the most severe lung disease on computed tomography, obstructive pulmonary function (83\%), and hypoxia/arterial hypoxaemia (67\%). The correlation between computed tomography and right heart abnormalities was significant ( $\chi^{2}$ analysis), $p=0.03$, table 3 . The correlation between spirometry and right heart abnormalities was highly significant $\mathrm{p}=0.007$, fig 2 . However the correlation between oxygen status and echo findings was not significant. Five of the six were male but this did not reach significance when compared with those without right heart abnormalities.

\section{DISCUSSION}

Primary antibody deficiency syndromes are uncommon but are associated with significant morbidity. Of these CVID is the most frequent, with an estimated incidence of one in 10000 to one in $50000 .^{2}$ Patients tend to present with recurrent respiratory tract infections so that chronic pulmonary complications are common, especially when there has been diagnostic delay. However CVID is a heterogeneous disorder which in some patients also includes cellular immune deficiency, so that disease phenotypes vary. Immunoglobulin replacement therapy can reduce the frequency of acute infections and improve lung function when trough IgG levels are maintained above $5 \mathrm{~g} / \mathrm{l} .{ }^{8}$ The optimal trough IgG level is not well established in CVID. A recent 


\begin{tabular}{|c|c|c|c|c|c|}
\hline $\begin{array}{l}\text { Patient } \\
\text { No }\end{array}$ & $\begin{array}{l}\text { Age in } \\
\text { years }\end{array}$ & Sex & $\begin{array}{l}\text { Diagnosis } \\
\text { date }\end{array}$ & $\begin{array}{l}\text { Estimated diagnostic } \\
\text { delay (years) }\end{array}$ & $\begin{array}{l}\text { Time on IgG } \\
\text { pre-echo (years) }\end{array}$ \\
\hline $1 \dagger$ & 70.4 & M & May 79 & 15 & 22.0 \\
\hline $2 \dagger$ & 49.4 & M & April 89 & 10 & 12.0 \\
\hline $3 \dagger$ & 53.75 & M & October 97 & 11 & 3.5 \\
\hline $4 \dagger$ & 49.1 & M & May 81 & 20 & 20.25 \\
\hline $5+$ & 48.5 & $\mathrm{~F}$ & March 81 & 7 & 20.4 \\
\hline $6^{*}+$ & 55.3 & $M$ & May 93 & 35 & 8.0 \\
\hline 7 & 61.6 & $\mathrm{~F}$ & March 82 & 20 & 13.8 \\
\hline 8 & 37.4 & $M$ & March 98 & 5 & 3.5 \\
\hline 9 & 69.0 & $\mathrm{~F}$ & 1961 & N/A & 35.0 \\
\hline 10 & 51.25 & $M$ & 1974 & 6 & 27.0 \\
\hline 11 & 57.9 & $M$ & 1972 & 10 & 29.0 \\
\hline 12 & 51.6 & $M$ & September 97 & 3 & 4.25 \\
\hline 13 & 35.5 & $M$ & October 99 & 3 & 1.8 \\
\hline 14 & 31.8 & $\mathrm{~F}$ & September 97 & 5 & 4.0 \\
\hline 15 & 49.0 & M & April 81 & 4 & 20.0 \\
\hline 16 & 77.25 & $\mathrm{~F}$ & January 94 & 14 & 7.5 \\
\hline 17 & 20.5 & $\mathrm{~F}$ & August 99 & 10 & 2.0 \\
\hline 18 & 36.6 & $\mathrm{~F}$ & 1986 & N/A & 15.0 \\
\hline 19 & 23.1 & $\mathrm{~F}$ & October 94 & & 7.0 \\
\hline 20 & 49.8 & M & 1981 & N/A & 20.0 \\
\hline \multicolumn{6}{|c|}{$\begin{array}{l}\text { N/A, data not available. } \\
\text { *Patient with } \lg G_{2} \text { subclass deficiency. } \\
\text { tPatients with right heart dilatation and/or moderate pulmonary hypertension. }\end{array}$} \\
\hline
\end{tabular}

study of pulmonary abnormalities in patients with primary hypogammaglobulinaemia (including 18 with CVID) has shown that pulmonary abnormalities develop in most patients and that silent progression can occur despite "adequate" replacement therapy with trough levels of $5 \mathrm{~g} / \mathrm{l}$ or more. ${ }^{9}$ Studies in patients with an X-linked primary antibody deficiency syndrome, X-linked agammaglobulinaemia, suggest that trough IgG levels $>8 \mathrm{~g} / \mathrm{l}$ can prevent the onset of bronchiectasis, chronic sinusitis, and non-bacterial infections if treatment is instituted early. ${ }^{10}$ On the basis of the latter study we aim for a trough IgG level above $8 \mathrm{~g} / \mathrm{l}$ in all of our antibody deficient patients, acknowledging that break-

Table 2 Positive echocardiographic findings in 20 patients with primary antibody deficiency

\begin{tabular}{|c|c|}
\hline $\begin{array}{l}\text { Patient } \\
\text { No }\end{array}$ & Positive echocardiographic findings \\
\hline 1 & $\begin{array}{l}\text { Mild left ventricular dilatation and hypertrophy with moderate } \\
\text { contraction, dilated aortic root and left atrium, mild mitral } \\
\text { regurgitation + tricuspid regurgitation, PAP } 41 \mathrm{~mm} \mathrm{Hg}\end{array}$ \\
\hline 2 & Mild LVH, mild right heart dilatation \\
\hline 3 & $\begin{array}{l}\text { Right heart dilatation, right atrial pressure } 18 \mathrm{~mm} \mathrm{Hg} \text {, dilated } \\
\text { hepatic veins, presumed moderate pulmonary hypertension, } \\
\text { unable to measure PAP }\end{array}$ \\
\hline 4 & Mild right heart dilatation \\
\hline 5 & PAP $48 \mathrm{~mm} \mathrm{Hg}$ \\
\hline 6 & $\begin{array}{l}\text { Dilated aortic root, moderate left ventricular contraction, right } \\
\text { atrial dilatation, PAP } 44 \mathrm{~mm} \mathrm{Hg}\end{array}$ \\
\hline 7 & Mild tricuspid regurgitation \\
\hline 8 & Mild tricuspid regurgitation, PAP $28 \mathrm{~mm} \mathrm{Hg}$ \\
\hline 9 & Mild LVH, mildly dilated left atrium \\
\hline 10 & Normal \\
\hline 11 & Mild LVH \\
\hline 12 & Mildly dilated aortic root \\
\hline 13 & Normal \\
\hline 14 & PAP $28 \mathrm{~mm} \mathrm{Hg}$ \\
\hline 15 & Normal \\
\hline 16 & $\begin{array}{l}\text { Anterior pericardial effusion }<1 \mathrm{~cm} \text {, moderate left ventricular } \\
\text { contraction, PAP } 33 \mathrm{~mm} \mathrm{Hg}\end{array}$ \\
\hline 17 & Normal \\
\hline 18 & Mild mitral regurgitation, PAP $29 \mathrm{~mm} \mathrm{Hg}$ \\
\hline 19 & PAP $26 \mathrm{~mm} \mathrm{Hg}$ \\
\hline 20 & Normal \\
\hline
\end{tabular}

LVH, left ventricular hypertrophy; PAP, mean pulmonary artery pressure. through infections can still occur and require early antibiotic therapy. ${ }^{11}$

Pulmonary hypertension is the major cardiovascular complication of obstructive lung disease. Hypoxaemia is associated with pathological changes that predominantly affect the peripheral blood vessels. Small arteries accumulate vascular smooth muscle cells in the intima and medial hypertrophy occurs in muscular pulmonary arteries. Structural change therefore, not simply hypoxic vasoconstriction, is the major factor in development of sustained pulmonary hypertension. ${ }^{12}$ Other contributory mechanisms may include hypercapnia, acidaemia, airway resistance, and endothelial derived vasoactive factors. ${ }^{12}$ Development of pulmonary hypertension is thought to adversely affect prognosis in obstructive lung disease, but whether this is a primary effect or simply a reflection of the severity of the underlying pulmonary disease is unclear. Treatment options for secondary pulmonary hypertension are limited, but include long term oxygen therapy, treatment of right heart failure and in some cases, anticoagulation. ${ }^{13}$ More recently, inhaled iloprost and oral bosentan (a dual endothelin receptor antagonist) have undergone clinical trials in severe pulmonary hypertension, both primary and secondary to connective tissue disease. ${ }^{14} 15$ Their place in the management of pulmonary hypertension secondary to obstructive lung disease, however, remains to be established.

Pulmonary hypertension and cor pulmonale are well documented complications of cystic fibrosis. This was used as a model disease for our current study as recurrent pulmonary infection and bronchiectasis are common to both cystic fibrosis and antibody deficiency syndromes. In 1980, Lester et al suggested a scoring system of echocardiographic abnormalities in patients with cystic fibrosis that correlated with clinical, chest imaging and pulmonary function scores. ${ }^{3}$ It has subsequently been shown that subclinical right ventricular dysfunction correlates with the severity of lung disease in cystic fibrosis. ${ }^{4}$ In addition, right ventricular overload can distort left ventricular diastolic function and left ventricular diastolic impairment closely correlates with pulmonary hypertension levels in chronic cor pulmonale. ${ }^{16}{ }^{17}$ Aortic root dilatation has also been described as in our cohort, ${ }^{3}$ but the clinical significance of this is not well established. 
Table 3 Spirometry, oxygen status, and thoracic computed tomography findings in 20 patients with primary antibody deficiency

\begin{tabular}{llll}
\hline Patient No & $\begin{array}{l}\text { Spirometry } \\
\text { FEV }_{1} / \text { FVC (\%) }\end{array}$ & Oxygen status* & Thoracic computed tomography \\
\hline $1 \dagger$ & $0.5 / 2.7(19)$ & 8.3 & Stable multilobar bronchiectasis \\
$2 \dagger$ & $1.0 / 2.4(42) \ddagger$ & $90 \%$ & Stable multilobar bronchiectasis \\
$3 \dagger$ & $0.6 / 2.9(21)$ & 10.5 & Severe emphysema \\
$4 \dagger$ & $3.1 / 5.7(54)$ & 10.0 & Stable single lobe bronchiectasis + emphysema \\
$5 \dagger$ & $2.7 / 3.5(77)$ & 13.4 & Normal \\
$6 \dagger$ & $0.9 / 1.55(58) \ddagger$ & $84 \%$ & Multilobar severe bronchiectasis \\
7 & $1.66 / 2.5(66) \ddagger$ & $96 \%$ & No residual bronchiectasis $\$$ \\
8 & $4.5 / 6.0(75)$ & 10.8 & Mild progressive bronchiectasis in left base \\
9 & $1.2 / 2.3(52)$ & 9.0 & Mild multilobar bronchiectasis \\
10 & $3.64 / 5.75(63) \ddagger$ & $95 \%$ & No significant bronchiectasis \\
11 & $3.4 / 4.9(69)$ & 12.0 & Reduced volume in left lower lobe \\
12 & $3.8 / 5.0(76)$ & N/A & Right basal scarring: not HRCT \\
13 & $2.7 / 4.0(68) \ddagger$ & $98 \%$ & Multilobar bronchiectasis \\
14 & $0.93 / 1.08(86) \ddagger$ & $95 \%$ & $?$ Alveolitis \\
15 & $3.8 / 4.9(78)$ & 10.3 & Mild multilobar bronchiectasis \\
16 & $\mathrm{~N} / \mathrm{A}$ & 9.14 & Stable multilobar bronchiectasis \\
17 & $2.76 / 2.95(94)$ & $96 \%$ & Normal \\
18 & $4.7 / 5.8(81)$ & 12.6 & Normal \\
19 & $1.95 / 1.95(100)$ & N/A & Normal \\
20 & $3.0 / 3.7(81)$ & N/A & Stable multilobar bronchiectasis \\
\hline
\end{tabular}

HRCT, high resolution computed tomography

*Figures with \% value measured by oximetry, figures without \% represent arterial oxygen pressure in $\mathrm{kPa}$. †Patients with right heart dilatation and/or moderate pulmonary hypertension.

$\ddagger$ Patient with formal pulmonary function tests within the last two years.

§lobectomy for bronchiectasis before antibody deficiency diagnosis.

Bronchiectasis was identified in $50 \%$ of the patients in this study, multilobar in $80 \%$ of these cases. We were interested to document the effect of such chronic lung disease on cardiac function in primary antibody deficiency.

Overall cardiac abnormalities, including secondary pulmonary hypertension, were common. There was no correlation between current age nor age at presentation with echo abnormalities; age itself therefore does not appear to be a significant determining factor. Left ventricular function was generally good in the absence of severe pulmonary hypertension but impaired function was observed in three patients, all of whom had evidence of hypoxaemia. When concentrating on right heart dilatation and/or at least moderate pulmonary hypertension, $30 \%$ of the patients were affected. Those with the most significant cardiac abnormalities had greater diagnostic delay $(\mathrm{p}=0.04)$, the most severe structural lung

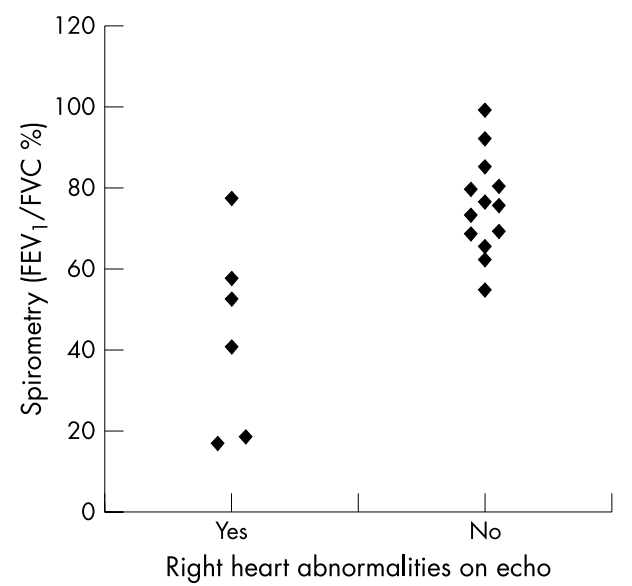

Figure 2 Correlation between spirometry and right heart abnormalities. The correlation between the spirometric values and right heart abnormalities is clearly demonstrated. Those patients with right heart abnormalities (defined as right heart dilatation and/or the presence of moderate pulmonary hypertension) have the most severely reduced lung function as measured by $\mathrm{FEV}_{1} / \mathrm{FVC}$. disease $(p=0.03)$, the worst pulmonary function $(p=0.007)$, and hypoxaemia, as postulated. However hypoxaemia was not significantly correlated with the presence of pulmonary hypertension, consistent with previous findings that hypoxia is not the single driving force. ${ }^{12}$ Neither were echo abnormalities simply a function of disease duration in our cohort. The delay in recognition of antibody deficiency, the adequacy of replacement immunoglobulin, and the access to antibiotics for intercurrent infection are all likely factors contributing to the degree of lung damage.

One patient (patient 5) had a normal thoracic computed tomogram within the previous five years and at the time of testing was not hypoxic. She had the highest recorded pulmonary artery pressure, which may therefore represent primary rather than secondary pulmonary hypertension. This is currently under further investigation.

Two of the six patients defined as having right heart abnormalities had peripheral oedema, treated with diuretics, but no other signs of right heart failure. No other patients had apparent clinical signs, supporting the concept of subclinical heart disease as seen in cystic fibrosis. Dyspnoea is a common symptom of pulmonary hypertension, bronchiectasis, and impaired left ventricular function so it is difficult to ascertain whether our patients had truly asymptomatic right heart abnormalities. Electrocardiographic data were available only for two of six patients with right heart abnormalities, one being normal and one showing partial right bundle branch block. We therefore have insufficient data to correlate electrocardiographic and echo findings. Smoking histories were not taken into account in this study. We advise all of our antibody deficient patients not to smoke. In view of this and the prolonged diagnostic delay in many cases, any such retrospective information is likely to be inaccurate and therefore of doubtful utility.

Despite the small patient numbers, our findings support the hypothesis that those with the most severe lung disease have the most significant cardiac abnormalities. This has not been previously documented in CVID. There is no reason to suspect that patients with primary antibody deficiency should behave any differently in terms of cardiac complications than patients with other chronic obstructive pulmonary 
disorders. We wish to emphasise that antibody deficiency is treatable and if recognised early does not have to lead to inexorable pulmonary damage that otherwise results in secondary pulmonary hypertension. Early recognition and adequate replacement therapy, probably aiming for trough IgG levels of $8 \mathrm{~g} / \mathrm{l}$ or above, may prevent progressive lung disease and hence cardiac manifestations. The optimal trough IgG level requires further prospective study, as our interpretation of "adequate" therapy is different from that of Kainulainen et al. ${ }^{9}$ This is likely to impact not only on lung disease but also on the availability of a scarce therapeutic resource.

We conclude that echocardiography does have a role in the evaluation of patients with primary antibody deficiency syndromes who present late with established bronchiectasis. Clinical signs of right ventricular dysfunction are often lacking. Dyspnoea may reflect secondary pulmonary hypertension in addition to the structural lung damage. Confirmation of pulmonary hypertension allows additional therapeutic options to be explored such as long term oxygen therapy.

\section{Authors' affiliations}

S L Johnston, R J Lock, D J Unsworth, M M Gompels, Department of Immunology and Immunogenetics, Southmead Hospital, Bristol, UK S J Hill, Department of Cardiology, Southmead Hospital, Bristol, UK J F Dwight, Department of Cardiology, John Radcliffe Hospital, Oxford, UK

\section{REFERENCES}

1 Spickett GP, Chapel HM. Report on the audit of patients with primary antibody deficiency in the United Kingdom 1993-1996. Newcastle upon Tyne: Newcastle upon Tyne Hospitals NHS Trust, 1998.
2 Report of an IUIS Scientific Committee. Primary immunodeficiency diseases. Clin Exp Immunol 1999;1 18(suppl 1): 1-28.

3 Lester LA, Egge AC, Hubbard VS, et al. Echocardiography in cystic fibrosis: a proposed scoring system. J Pediatr 1980;97:742-8.

4 lonescu AA, lonescu A-A, Payne N, et al. Subclinical right ventricular dysfunction in cystic fibrosis. A study using tissue Doppler echocardiography. Am J Respir Crit Care Med 2001;163:1212-18.

5 Vizza CD, Lynch JP, Ochoa LL, et al. Right and left ventricular dysfunction in patients with severe pulmonary disease. Chest 1998; 113:576-83.

6 Walsh C, Wilde P. Practical echocardiography. 1 st Ed. London: Greenwich Medical Media Limited, 1999 (distributed worldwide by Oxford University Press).

7 Official Statement of the European Respiratory Society. Standardized lung function testing. Eur Respir J Suppl 1993;16:1-100.

8 Roifman CM, Levison H, Gelfand EW. High-dose versus low-dose intravenous immunoglobulin in hypogammaglobulinaemia and chronic lung disease. Lancet 1987;i:1075-7.

9 Kainulainen L, Varpula M, Liippo K, et al. Pulmonary abnormalities in patients with primary hypogammaglobulinemia. J Allergy Clin Immunol 1999;104:1031-6.

10 Quartier P, Debré M, De Blic J, et al. Early and prolonged intravenous immunoglobulin replacement therapy in childhood agammaglobulinemia: a retrospective survey of 31 patients. J Pediatr 1999;134:589-96.

11 Pettit SJ, Bourne H, Spickett GP. Survey of infection in patients receiving antibody replacement treatment for immune deficiency. J Clin Pathol 2002;55:577-80.

12 MacNee W. Pathophysiology of cor pulmonale in chronic obstructive pulmonary disease. Am J Respir Crit Care Med 1994;150:833-52.

13 Ricciardi MJ, Rubenfire M. How to manage secondary pulmonary hypertension. Recognising and treating cor pulmonale and chronic thromboembolism. Postgrad Med 1999; 105:183-90.

14 Olschewski H, Simonneau G, Galiè N, et al. Inhaled iloprost for severe pulmonary hypertension. N Engl J Med 2002;347:322-9.

15 Rubin LJ, Badesch DB, Barst RJ, et al. Bosentan therapy for pulmonary arterial hypertension. N Engl J Med 2002;346:896-903.

16 Tutar E, Kaya A, Güleç S, et al. Echocardiographic evaluation of left ventricular diastolic function in chronic cor pulmonale. Am J Cardiol 1999;83:1414-17.

17 Schena M, Clini E, Errera D, et al. Echo-Doppler evaluation of left ventricular impairment in chronic cor pulmonale. Chest 1996;109:1446-51. 